\title{
Death After Discharge: Prognostic Model of 1-year Mortality in Traumatic Brain Injury Patients Undergoing Decompressive Craniectomy
}

\section{Wenxing Cui}

Tangdu Hospital

Shunnan Ge

Tangdu Hospital

Yingwu Shi

Tangdu Hospital

Xun Wu

Jianing Luo

Tangdu Hospital

Haixiao Lui

Tangdu Hospital

Gang Zhu

Tangdu Hospital

Hao Guo

Dayun Feng

Tangdu Hospital

Yan Qu ( $D$ yanqu0123@fmmu.edu.cn )

Tangdu Hospital

\section{Research}

Keywords: Decompressive craniectomy, Traumatic brain injury, 1-year mortality, Prognostic model, Random forest

Posted Date: September 4th, 2020

DOI: https://doi.org/10.21203/rs.3.rs-70888/v1

License: (c) (1) This work is licensed under a Creative Commons Attribution 4.0 International License. Read Full License 
Version of Record: A version of this preprint was published at Chinese Neurosurgical Journal on April 21st, 2021. See the published version at https://doi.org/10.1186/s41016-021-00242-4. 


\section{Abstract}

OBJECTIVE: Despite advances in decompressive craniectomy (DC) for the treatment of traumatic brain injury (TBI), a high risk of poor long-term prognosis exists in these patients. The aim of this study is to predict 1-year mortality in TBI patients undergoing DC using the logistic regression and random tree models.

METHODS: This was a retrospective analysis of TBI patients undergoing DC from January 1, 2015 to April 25, 2019. Patient demographic characteristics, biochemical tests and intraoperative factors were collected. 1-year mortality prognostic models were developed using multivariate logistic regression and random tree algorithms. Overall accuracy, sensitivity, specificity and area under the receiver operating characteristic curves (AUC) were used to evaluate model performance.

RESULTS: Of the 230 patients, 70 (30.4\%) died within 1 year. Older age (OR, 1.066; 95\% Cl, 1.045-1.087; P $<0.001)$, higher Glasgow coma score (GCS) (OR, 0.737; 95\% Cl, 0.660-0.824; $\mathrm{P}<0.001)$, higher d-dimer (OR, 1.005; 95\% Cl, 1.001-1.009; $\mathrm{P}=0.015)$, coagulopathy (OR, 2.965; 95\% Cl, 1.808-4.864; $\mathrm{P}<0.001$ ), hypotension (OR, 3.862; 95\% Cl, 2.176-6.855; $\mathrm{P}<0.001)$ and completely effaced basal cisterns $(\mathrm{OR}, 3.766$; $95 \% \mathrm{Cl}, 2.255-6.290 ; \mathrm{P}<0.001)$ were independent predictors of 1-year mortality. Random forest demonstrated better performance for 1-year mortality prediction, which achieved overall accuracy of 0.810 , sensitivity of 0.833 , specificity of 0.800 , and AUC of 0.830 on the testing data, compared to logistic regression model.

CONCLUSIONS: Random forest model showed relatively good predictive performance for 1-year mortality in TBI patients undergoing DC. Further external test is required to verify our prognostic model.

\section{Introduction}

TBI is a common cause of death and disability worldwide affecting all age groups ${ }^{1}$. DC, surgically removing a component of the skull, has been executed in TBI patients for many years ${ }^{2,3}$, especially for severe patients with high intracranial pressure $(\mathrm{ICP})^{4}$, which can effectively increase cerebral perfusion pressure. Two randomized clinical trials, DECRA ${ }^{5}$ and RESCUEicp ${ }^{6}$ trials, have been conducted with respect to the prognosis of TBI patients after DC. DECRA demonstrated that early bifronto-temporoparietal DC decreased the length of stay in the ICU but was associated with unfavorable outcomes; RESCUEicp reported that DC in patients with TBI and refractory intracranial hypertension led to lower mortality.

In the previous research, the short-term outcome predictive scoring models, discharge status ${ }^{7}$ and 30 -day mortality ${ }^{8}$, were well established in TBI patients with DC. However, long-term mortality predictive model is also a vital issue which has not gained well-deserved attention. Despite of several studies on long-term outcomes, such as, predictors of 1-year mortality in older brain-injured patients ${ }^{9}$, functional outcomes from 3 to 24 months following severe brain injury ${ }^{10}$, the study population was not specifically targeted at 
patients after DC. It is necessary to identify the predictors of long-term mortality in TBI patients after DC, which contributed to the understanding of disease progression, thereby providing better daily care to improve the quality of life of patients.

Machine learning has been widely used in disease diagnosis and prognosis prediction ${ }^{11,12}$. For example, machine learning was applied to predict in-hospital morbidity and mortality after TBI, which demonstrated the relatively good predictive performance ${ }^{13}$. So far the exploration of machine learning techniques to predict the long-term prognosis of TBI patients after DC has been rarely done. Thus, the purpose of this study was to develop prognostic models to predict 1-year mortality of TBI patients undergoing $D C$ by using the logistic regression and random tree models.

\section{Method}

\section{Patient population}

This retrospective study was approved by the ethical committee of Tangdu Hospital, Fourth Military Medical University. We reviewed consecutive 947 TBI patients treated at Tangdu Hospital from January 1, 2015 to April 25, 2019. Our main inclusion criteria covered patients who underwent DC with a history of TBI. The main indications and approach were described as previous studies ${ }^{14,15}$. The exclusion criteria were listed as follows: (1) the interval from injury to admission was more than 24 hours; (2) died in the hospital; (3) other severe systemic diseases, such as malignant tumors, cirrhosis and uremia; (4) lost to follow-up.

\section{Variables And Data Collection}

The following data were extracted from the registry database by five study nurse: patient demographic characteristics; GCS score; biochemical tests including aPTT, INR, platelet counts, d-dimer, fibrinogen, glucose, red blood cell and neutrophil/lymphocytes ratio (NLR) the initial CT scan characteristics including contusion volume, subarachnoid hemorrhage (Fisher scales), midline shift and basal cistern status; perioperative bleeding; neurologic worsening conditions including mechanical ventilation, tracheotomy, deep venous thrombosis and hypotension which needed noradrenaline to correct. INR, aPTT and platelet counts were used to define the traumatic coagulopathy, according to the previous study ${ }^{16}$, aimed at simplifying the prognostic model. Coagulopathy was defined as an aPTT > $36 \mathrm{sec}$ and/or a PT in INR $>1.2$, and/or a platelet count $<100 \times 10^{9}$ per liter.

\section{Prognostic Model}

Of the total 230 patients, 172 patients (75\%) were randomly selected for training and the remaining 58 patients $(25 \%)$ for testing. The random seed was set as 66511 . The ratio of non-survivor to survivor was 1:2, so SMOTE (Synthetic Minority Oversampling Technique) was used to balance the training data. We 
conducted a performance comparison of the logistic regression and random tree models. The 10-fold cross-validation, repeated three times, was done by using the original $75 \%$ of the data treated as training data. Features were selected by using the univariate logistic regression method. Hyperparameter optimization was achieved by the grid search method. Three parameters were determined for random tree model: "Gini" impurity criterion; $m$ try $=4$; tree $=100$. An open-source programming language R 3.6.1 and an efficient machine learning tool GraphLab Create were used for machine learning coding.

We compared the predictive performance of the logistic regression and random tree models according to accuracy, sensitivity, specificity, and AUC. The meaning of accuracy, sensitivity and specificity was described as the previous study ${ }^{17}$.

\section{Statistical Analysis}

Categorical variables were expressed as the frequency (percentages), and continuous variables with skewed distributions were presented as the median and interquartile range (IQR). Univariate logistic regression was used to find significant predictive variables $(P<0.05)$, which were entered into the multivariate regression by using the Forward:LR method to determine the independent risk factors for 1year mortality. A nomogram model was developed to predict the probability of 1-year mortality. All data were analyzed with statistical software SPSS 20.0 (IBM, New York, NY).

\section{Result}

\section{Patient population}

Of the 947 patients treated in this research center during the study period, 306 patients who underwent DC were identified. According to our inclusion and exclusion criteria, 230 patients were enrolled at last. By February 26, 2020, 89 (38.7\%) patients had died, 141 (61.3\%) had survived. The survival analysis showed that the 3-month survival rate was $0.826,6$-month survival rate was $0.774,1$-year survival rate was 0.695 and the 3-year survival rate was 0.623 (Fig. 1).

The patient demographic and clinical characteristics are summarized in Table 1.70 patients $(30.4 \%)$ died within one year who underwent DC. The median age was 59 years (IQR, 50-65), and 16 (22.86\%) patients were female. In this study, the most frequent mechanism of injury was the motor vehicle accident (114 patients; $49.557 \%$ ); Falling were also common (82 patients; $36.65 \%$ ). The median GCS score at admission was 4.5 (IQR, 3-7). The total biochemical tests were as follows. Fibrinogen was $1.86 \mathrm{~g} / \mathrm{L}$ (IQR, 1.392.27). D-dimer was $42.56 \mathrm{mg} / \mathrm{mL}$ (IQR, 18.47-98.10). 51 (22.17\%) patients had coagulopathy disorders. Abnormal glucose $(>8.33 \mathrm{mmol} / \mathrm{L})$ and red blood cell $\left(\mathrm{man}>5.5 \times 10^{12} / \mathrm{L}\right.$ or $<4.0 \times 10^{12} / \mathrm{L}$, woman $>5.0$ $\times 10^{12} / \mathrm{L}$ or $<3.5 \times 10^{12} / \mathrm{L}$ ) were shown in $159(69.13 \%)$ patients and $56(24.56 \%)$ patients, respectively. $\mathrm{N} / \mathrm{L}$ ratio was 14.55 (IQR, 8.29-24.57). In the initial CT scan characteristics, contusion volume was $15.99 \mathrm{~cm}^{3}$ (IQR, 0-40.82) and subarachnoid hemorrhage (Fisher scales) was 2 (0-3) in total. Midline shift and completely effaced basal cisterns was shown in 97 (42.17\%) patients and 40 (17.39\%) patients, 
respectively. Perioperative bleeding was more than $750 \mathrm{ml}$ shown in $178(77.39 \%)$ patients. Neurologic worsening conditions in total were listed as follows. Mechanical ventilation, tracheotomy, deep venous thrombosis and hypotension which needed noradrenaline to treat were showed in $84(36.52 \%)$ patients, $102(44.35 \%)$ patients, $22(9.57 \%)$ patients, 39 (16.96\%) patients, respectively.

\section{Prognostic factors predicting 1-year mortality in the Univariate and Multivariate Analyses}

Univariate analyses of the relationship between clinical variables and 1-year mortality are shown in Table 1. Age $(P<0.001)$, lower GCS $(P<0.001)$, higher $d$-dimer $(P<0.001)$, coagulopathy $(P=0.027)$, hypotension $(P=0.021)$, completely effaced basal cisterns $(P=0.004)$ and perioperative bleeding > $750 \mathrm{ml}(P=0.049)$ were associated with 1-year mortality. Table 2 lists the results of the multivariate regression analysis to predict 1-year mortality. Older age $(P<0.001)$, lower GCS $(P<0.001)$, higher d-dimer $(P=0.015)$, coagulopathy $(P<0.001)$, hypotension $(P<0.001)$ and completely effaced basal cisterns $(P=$ $0.004)$ were independent predictors of 1 -year mortality.

Next, we incorporated all independent predictors identified in multivariate regression analysis to create nomograms, as is shown in Fig. 2. The nomogram was constructed by settling a score to each parameter with a point range from 0 to 100 . Summing the points arranged to each predictor can generate the total score, which converted into an individual probability of 1-year mortality (from 1-99\%) finally. Based on the condition of patients, this nomogram can predict 1-year mortality simply and intuitively.

\section{Prediction Performance Of Logistic Regression And Random Tree Models}

To identify the importance of each predictors for 1-year mortality, we chose the feature selection method by applying the random forest algorithm. It has been shown that three parameters are the most important for predicting 1-year mortality: age, GCS, and d-dimer (Fig. 3). Interestingly, perioperative bleeding was identified as the last associated factor for 1-year mortality.

Their ROC and AUC were calculated to evaluate their discriminative ability (Fig. 4). To evaluate the prediction performance of Logistic Regression and Random Tree models, the five-fold cross-validation was done on the training data (Table 3). Before SMOTE used to balance the training data, we developed the logistic regression model. On the training data, it achieved overall accuracy of 0.750 , sensitivity of 0.731 , specificity of 0.758 and AUC of 0.770 when at the optimal cut-off point (0.307); on the testing data, it achieved an overall accuracy of 0.672 , sensitivity of 1.000 , specificity of 0.525 and AUC of 0.765 when at the optimal cut-off point (0.195). Next, we balanced the training data by using SMOTE. In the logistic regression model, it achieved an overall accuracy of 0.756 , sensitivity of 0.615 , specificity of 0.817 and AUC of 0.760 on the training data, while an overall accuracy of 0.741 , sensitivity of 0.889 , specificity of 0.675 and AUC of 0.843 on the testing data, at the optimal cut-off point, respectively. In the random forest model, it achieved an overall accuracy of 0.983 , sensitivity of 1.000 , specificity of 0.975 and AUC of 0.998 on the training data, while an overall accuracy of 0.810 , sensitivity of 0.833 , specificity of 0.800 and AUC 
of 0.830 on the testing data, at the optimal cut-off point, respectively. The accuracy, sensitivity, specificity, and AUC at cut-off point (0.5) of three prognostic models were shown in Table 3.

\section{Discussion}

In our study, we found that older age, lower GCS, higher d-dimer, coagulopathy, hypotension and completely effaced basal cisterns were independent predictors of 1-year mortality in patients with TBI after DC. Compared to the logistic regression model, the random tree model presented a better performance with respect to accuracy, sensitivity, specificity and AUC on the training data (Whether the cut-off point was 0.5 or the optimal point). So did the random tree model in regard to accuracy, sensitivity and specificity on the testing data, when the cut-off point was 0.5 . Although the sensitivity of the random tree model was inferior to that of logistic regression model on the testing data at the optimal cut-off point, the accuracy and specificity of the random tree model achieved better. The AUC of the two models is similar on the testing data. On the whole, this finding suggests that the random tree is a valuable and accurate model to predict 1-year mortality in TBI patients after DC. Additionally, our study chose the time of 1-year mortality based on the survival analysis of TBI patients undergoing DC, which showed that the mortality rate within 1 year after discharge was very high. Since we turned the spotlight on the long-term outcomes of TBI patients, patients who died in the hospital were excluded. The predictors of inpatient death and post-discharge mortality were disparate, as the previous study showed ${ }^{9}$. Thus, our study on 1year mortality, which excluded patients who died in the hospital, could show better predictive performance to some extent.

Age and GCS, which were already found as important predictors of TBI, were also confirmed in our study ${ }^{13,18}$. Tian et al. ${ }^{7}$ identified that age was one of the independent risk factors for discharge status after DC, and Tang et al. ${ }^{8}$ also observed that age was the risk factor for 30-day mortality after DC. Combined with our research, older age is a risk factor for both short-term and long-term outcomes of TBI patients after DC. Older people tend to suffer from many basic diseases, and the rehabilitation of the body was poorer after TBI than young people, as we know. GCS, which was similar to age, was also a powerful predictor for outcomes of TBI after $D C^{8,19}$. D-dimer, a degradation product of fibrinogen, reflected the fibrinolysis of the body. Many studies found that higher d-dimer at admission was associated with higher risk of progressive hemorrhagic injury ${ }^{20,21}$, while a meta-analysis about prognostic role of d-dimer level on admission in TBI patients found no significant relationship between $\mathrm{d}$ dimer and the risk of poor functional outcome at 3 months ${ }^{22}$. In our study, higher d-dimer was one of the predictors for 1-year mortality after DC. In our opinion, the prognostic role of $d$-dimer may be related to the study population and specific outcomes. It is considered that secondary coagulopathy after TBI is an important factor for unfavorable outcome ${ }^{23,24}$, and our results also confirmed this finding. TBI-induced coagulopathy is very common, ranging from $7-54 \% 25,26$. Coagulopathy generated by TBI is a systemic manifestation of local injury ${ }^{27}$. Pro-coagulant vesicles (including tissue factors, cardiolipin, vWF, etc.) from damaged brain tissue are released into systemic circulation ${ }^{28-30}$, making the balance between coagulation and anticoagulation broken. This distinct pathogenetic pathway arises increasing attention, 
and how to intervene in this process is crucial for the prognosis of TBI patients. Hypotension was another risk factor for 1-year mortality. The prognostic role of hypotension in TBI was poorly elaborated. Tang et al. ${ }^{8}$ found that intraoperative hypotension was associated with 30-day mortality in TBI patients after DC. In our study, we recorded the incidence of hypotension throughout the course of the disease. Additionally, noradrenaline was injected to maintain vital signs in patients with hypotension. Our study suggests that hypotension is a crucial predictor in long-term prognosis that cannot be ignored in TBI patients after DC. However, some more specific questions between hypotension and the outcome of TBI need to be addressed. For example, whether the course of hypotension in patients with TBI is associated with the outcomes. And the risk factors underlying hypotension in TBI need to be explored. Completely effaced basal cistern status, which represents severe elevated ICP, was found as an important predictor of outcome in the previous study ${ }^{8,31}$. Basal cistern effacement is closely associated with pupillary reactivity midline shift. Thus, it can represent a uniquely useful neuroimaging characteristic to guide intervention in $\mathrm{TBl}^{32}$.

Many studies on TBI have been conducted using modern machine learning algorithm, owing to its good prediction performance. Matsuo et al. ${ }^{13}$ demonstrated that random forest showed good performance for poor outcome prediction at discharge and ridge regression for in-hospital mortality prediction in TBI, both of which achieved almost the accuracy of 0.9. Based on the feature selection method, age and GCS, in their study, appeared to be the most important predictors for both poor outcome and mortality, which was consistent with our findings. A total of 232 patients with TBI were included and separated into training data and test data, which was comparable with our samples of 230 patients. The prediction of mortality was better than our results, which were 0.886 accuracy and 0.875 AUC on the testing data. The difference in performance is mainly due to the prediction of death at different times, and there is no doubt that longterm mortality is harder to predict than in-hospital mortality. Rughani et al. ${ }^{33}$ used the artificial neural network to predict in-hospital survival of TBI patients, which achieved the accuracy of 0.878 and the AUC of 0.860 . They included 11 variables in the model: age, sex, total GCS score, and individual components of the GCS score at the scene of injury and emergency department, and first systolic blood pressure. Nonetheless, some vital parameters, such as biochemical tests, CT scan characteristics and neurologic worsening conditions, were absent in their model. Although one study predicted 18-month mortality in severe TBI after DC using the IMPACT prognostic model ${ }^{19}$, whose AUC was 0.77 , our random tree prediction model achieved the AUC of 0.998 and 0.830 on the testing and training data, respectively. This suggests that machine learning models perform better in outcome prediction than the traditional logistic regression models. At present, machine learning algorithm has been increasingly used in prognosis of $\mathrm{TBI}^{13,34,35}$, and it enables us to optimize the treatment strategy and provide better daily care.

\section{Limitations}

There are several limitations in our study. First of all, it was a single-center, retrospective, and nonrandomized study, in which there may exist selection bias. Next, we did not include ICP data in this study, based on the fact that ICP monitor was not performed on every patient in previous clinical work. 
Thus, we could not include this vital variable to avoid apparent selection bias. Another limitation is that some information about the patient after discharge, such as the rehabilitation therapy, was absent, but this provides an opportunity for prospective research to analyze this variable. Finally, our prognostic model mainly aimed at TBI patients undergoing DC, so the performance of our model may decrease when it is applied to all TBI patients.

\section{Conclusion}

Our findings confirm that older age, lower GCS, higher d-dimer, coagulopathy, hypotension and completely effaced basal cisterns were associated with 1-year mortality. Random forest model showed relatively good predictive performance for 1-year mortality, which achieved overall accuracy of 0.810 , sensitivity of 0.833 , specificity of 0.800 , and AUC of 0.830 on the testing data. Our results indicated that machine learning turned in a good performance for TBI outcome. By virtue of machine learning with more accurate prediction performance, we can provide TBI patients with better precision medicine and care direction.

\section{Abbreviations}

DC

decompressive craniectomy

$\mathrm{TBI}$

Traumatic brain injury

AUC

Area under receiver operating characteristic curves

GCS

Glasgow coma score

ICP

Intracranial pressure

\section{Declarations}

\section{Ethics approval and consent to participate}

This study approved by the ethical committee of Tangdu Hospital, Fourth Military Medical University (K201907-06), and the consent was waived.

\section{Consent for publication}

The consent for publication from patients was approved.

\section{Availability of data and material}

Please contact author for data requests. 


\section{Competing interests}

No competing interests exist for any of the authors.

\section{Funding}

This work was supported by grants from the Chang Jiang Scholar Program of China and the National Natural Science Foundation of China $(81630027,81571215)$.

\section{Authors' contributions}

WC, GS, and SY contributed equally to this work. All authors met ICMJE criteria for authorship. YQ, WC, $\mathrm{GS}$, and SY contributed to the conception and design. $\mathrm{XW}$, JL, and $\mathrm{HL}$ performed the statistical analysis. $\mathrm{GZ}, \mathrm{HG}$, and DF helped to draft the manuscript. All authors approved the final manuscript.

\section{Acknowledgments}

We thank Ping Wang and Yaning Cai for their help with development and maintenance of the neurotrauma database.

\section{References}

1. Capizzi A, Woo J, Verduzco-Gutierrez M. Traumatic Brain Injury: An Overview of Epidemiology, Pathophysiology, and Medical Management. The Medical clinics of North America. 2020;104(2):213-38. https://doi.org/10.1016/j.mcna.2019.11.001.

2. Guerra WK, Gaab MR, Dietz H, Mueller JU, Piek J, Fritsch MJ. Surgical decompression for traumatic brain swelling: indications and results. Journal of neurosurgery. 1999;90(2):187-96. https://doi.org/10.3171/jns.1999.90.2.0187.

3. Kwan K, Schneider J, Ullman JS. Chapter 12: Decompressive Craniectomy: Long Term Outcome and Ethical Considerations. Frontiers in neurology. 2019;10:876. https://doi.org/10.3389/fneur.2019.00876.

4. Bor-Seng-Shu E, Figueiredo EG, Amorim RL, et al. Decompressive craniectomy: a meta-analysis of influences on intracranial pressure and cerebral perfusion pressure in the treatment of traumatic brain injury. Journal of neurosurgery. 2012;117(3):589-96. https://doi.org/10.3171/2012.6.Jns101400.

5. Cooper DJ, Rosenfeld JV, Murray L, et al. Decompressive craniectomy in diffuse traumatic brain injury. The New England journal of medicine. 2011;364(16):1493-502. https://doi.org/10.1056/NEJMoa1102077.

6. Hutchinson PJ, Kolias AG, Timofeev IS, et al. Trial of Decompressive Craniectomy for Traumatic Intracranial Hypertension. The New England journal of medicine. 2016;375(12):1119-30. https://doi.org/10.1056/NEJMoa1605215. 
7. Tian R, Liu W, Dong J, et al. Prognostic Predictors of Early Outcomes and Discharge Status of Patients Undergoing Decompressive Craniectomy After Severe Traumatic Brain Injury. World Neurosurg. 2019;126:e101-8. https://doi.org/10.1016/j.wneu.2019.01.246.

8. Tang Z, Yang K, Zhong M, et al. Predictors of 30-Day Mortality in Traumatic Brain-Injured Patients after Primary Decompressive Craniectomy. World Neurosurg. 2020;134:e298-305. https://doi.org/10.1016/j.wneu.2019.10.053.

9. Peck KA, Calvo RY, Sise CB, et al. Death after discharge: predictors of mortality in older brain-injured patients. The journal of trauma acute care surgery. 2014;77(6):978-83. https://doi.org/10.1097/ta.0000000000000356.

10. Wilkins TE, Beers SR, Borrasso AJ, et al. Favorable Functional Recovery in Severe Traumatic Brain Injury Survivors beyond Six Months. J Neurotrauma. 2019;36(22):3158-63. https://doi.org/10.1089/neu.2018.6153.

11. Chen JH, Asch SM. Machine Learning and Prediction in Medicine - Beyond the Peak of Inflated Expectations. The New England journal of medicine. 2017;376(26):2507-9. https://doi.org/10.1056/NEJMp1702071.

12. Doupe P, Faghmous J, Basu S. Machine Learning for Health Services Researchers. Value in health: the journal of the International Society for Pharmacoeconomics Outcomes Research. 2019;22(7):808-15. https://doi.org/10.1016/j.jval.2019.02.012.

13. Matsuo K, Aihara H, Nakai T, Morishita A, Tohma Y, Kohmura E. Machine Learning to Predict InHospital Morbidity and Mortality after Traumatic Brain Injury. J Neurotrauma. 2020;37(1):202-10. https://doi.org/10.1089/neu.2018.6276.

14. Kolias AG, Kirkpatrick PJ, Hutchinson PJ. Decompressive craniectomy: past, present and future. Nature reviews Neurology. 2013;9(7):405-15. https://doi.org/10.1038/nrneurol.2013.106.

15. Mao X, Miao G, Hao S, et al. Decompressive craniectomy for severe traumatic brain injury patients with fixed dilated pupils. Therapeutics clinical risk management. 2015;11:1627-33. https://doi.org/10.2147/tcrm.S89820.

16. Lustenberger T, Talving P, Kobayashi L, et al. Early coagulopathy after isolated severe traumatic brain injury: relationship with hypoperfusion challenged. The Journal of trauma. 2010;69(6):1410-4. https://doi.org/10.1097/TA.0b013e3181cdae81.

17. Liu J, Xiong Y, Zhong M, et al. Predicting Long-Term Outcomes After Poor-Grade Aneurysmal Subarachnoid Hemorrhage Using Decision Tree Modeling. Neurosurgery. 2020;87(3):523-9. https://doi.org/10.1093/neuros/nyaa052.

18. Murray GD, Butcher I, McHugh GS, et al. Multivariable prognostic analysis in traumatic brain injury: results from the IMPACT study. J Neurotrauma. 2007;24(2):329-37. https://doi.org/10.1089/neu.2006.0035.

19. Honeybul S, Ho KM. Predicting long-term neurological outcomes after severe traumatic brain injury requiring decompressive craniectomy: A comparison of the CRASH and IMPACT prognostic models. Injury. 2016;47(9):1886-92. https://doi.org/10.1016/j.injury.2016.04.017. 
20. Yuan F, Ding J, Chen $\mathrm{H}$, et al. Predicting progressive hemorrhagic injury after traumatic brain injury: derivation and validation of a risk score based on admission characteristics. J Neurotrauma. 2012;29(12):2137-42. https://doi.org/10.1089/neu.2011.2233.

21. Tong WS, Zheng P, Zeng JS, et al. Prognosis analysis and risk factors related to progressive intracranial haemorrhage in patients with acute traumatic brain injury. Brain Inj. 2012;26(9):113642. https://doi.org/10.3109/02699052.2012.666437.

22. Zhang J, He M, Song Y, Xu J. Prognostic role of D-dimer level upon admission in patients with traumatic brain injury. Medicine. 2018;97(31):e11774. https://doi.org/10.1097/md.0000000000011774.

23. Sun $Y$, Wang J, Wu X, et al. Validating the incidence of coagulopathy and disseminated intravascular coagulation in patients with traumatic brain injury-analysis of 242 cases. British journal of neurosurgery. 2011;25(3):363-8. https://doi.org/10.3109/02688697.2011.552650.

24. Franschman G, Boer $C$, Andriessen TM, et al. Multicenter evaluation of the course of coagulopathy in patients with isolated traumatic brain injury: relation to $\mathrm{CT}$ characteristics and outcome. $\mathrm{J}$ Neurotrauma. 2012;29(1):128-36. https://doi.org/10.1089/neu.2011.2044.

25. Chhabra G, Rangarajan K, Subramanian A, Agrawal D, Sharma S, Mukhopadhayay AK. Hypofibrinogenemia in isolated traumatic brain injury in Indian patients. Neurol India. 2010;58(5):756-7. https://doi.org/10.4103/0028-3886.72175.

26. Greuters S, van den Berg A, Franschman G, et al. Acute and delayed mild coagulopathy are related to outcome in patients with isolated traumatic brain injury. Crit Care (London England). 2011;15(1):R2. https://doi.org/10.1186/cc9399.

27. Blood JZFZ. DJJ. Coagulopathy induced by traumatic brain injury: systemic manifestation of a localized injury. 2018;131(18): 2001-2006. https://doi.org/10.1182/blood-2017-11-784108.

28. Zhao Z, Wang M, Tian Y, et al. Cardiolipin-mediated procoagulant activity of mitochondria contributes to traumatic brain injury-associated coagulopathy in mice. Blood. 2016;127(22):276372. https://doi.org/10.1182/blood-2015-12-688838.

29. Wu Y, Liu W, Zhou Y, et al. von Willebrand factor enhances microvesicle-induced vascular leakage and coagulopathy in mice with traumatic brain injury. Blood. 2018;132(10):1075-84. https://doi.org/10.1182/blood-2018-03-841932.

30. Tian $Y$, Salsbery B, Wang M, et al. Brain-derived microparticles induce systemic coagulation in a murine model of traumatic brain injury. Blood. 2015;125(13):2151-9. https://doi.org/10.1182/blood2014-09-598805.

31. Kolias AG, Adams H, Timofeev I, et al. Decompressive craniectomy following traumatic brain injury: developing the evidence base. British journal of neurosurgery. 2016;30(2):246-50. https://doi.org/10.3109/02688697.2016.1159655.

32. Nourallah B, Menon DK, Zeiler FA. Midline Shift is Unrelated to Subjective Pupillary Reactivity Assessment on Admission in Moderate and Severe Traumatic Brain Injury. Neurocritical care. 2018;29(2):203-13. https://doi.org/10.1007/s12028-018-0526-8. 
33. Rughani Al, Dumont TM, Lu Z, et al. Use of an artificial neural network to predict head injury outcome. Journal of neurosurgery. 2010;113(3):585-90. https://doi.org/10.3171/2009.11.Jns09857.

34. Folweiler KA, Sandsmark DK, Diaz-Arrastia R, Cohen AS, Masino AJ. Unsupervised Machine Learning Reveals Novel Traumatic Brain Injury Patient Phenotypes with Distinct Acute Injury Profiles and Long-Term Outcomes. J Neurotrauma. 2020;37(12):1431-44. https://doi.org/10.1089/neu.2019.6705.

35. Hernandes Rocha TA, Elahi C, Cristina da Silva N, et al. A traumatic brain injury prognostic model to support in-hospital triage in a low-income country: a machine learning-based approach. Journal of neurosurgery 2019: 1-9. https://doi.org/10.3171/2019.2.Jns182098.

\section{Tables}

Due to technical limitations, table 1,2 \& 3 is only available as a download in the Supplemental Files section.

\section{Figures}




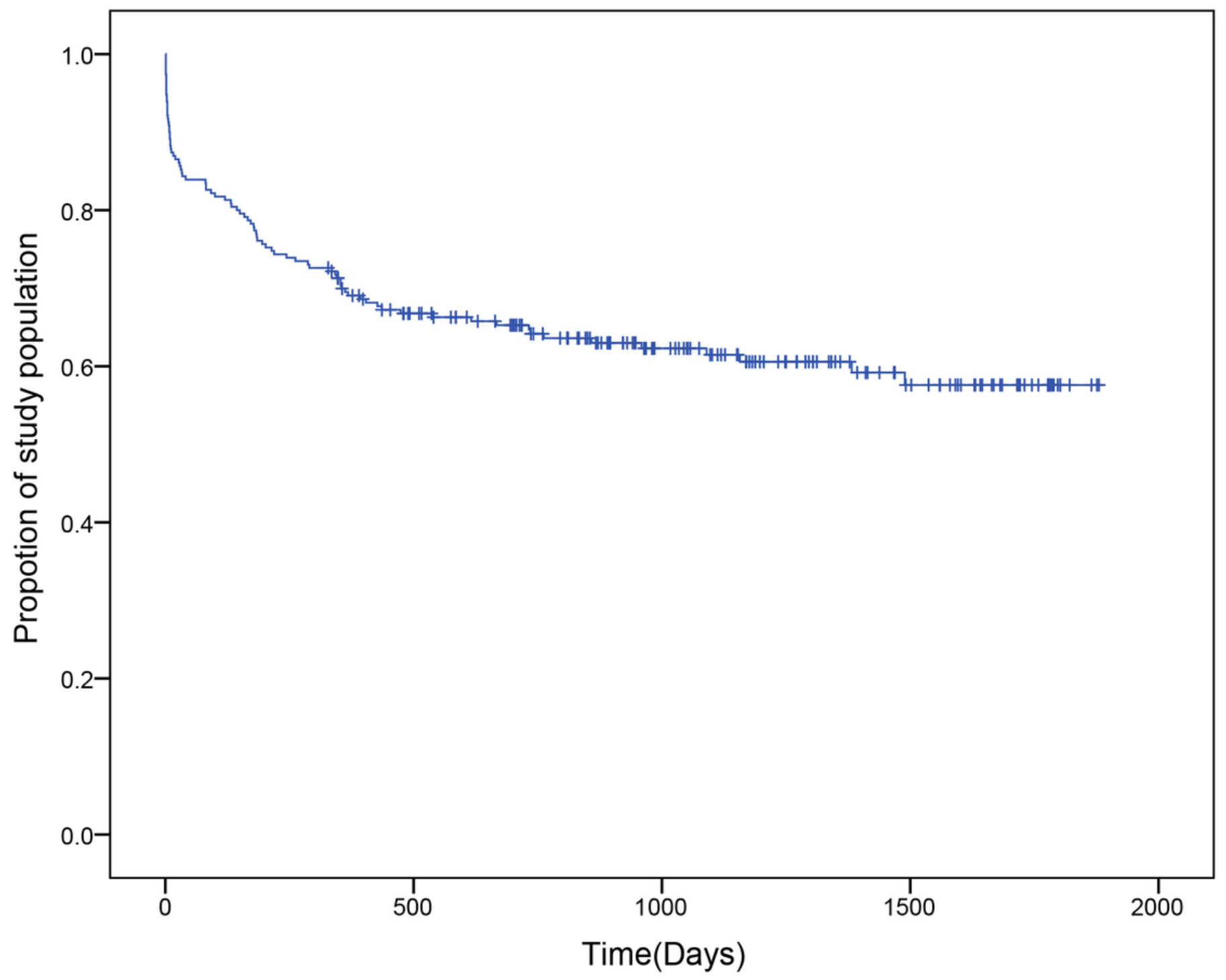

Figure 1

Kaplan-Meier survival curves for TBI patients undergoing DC 


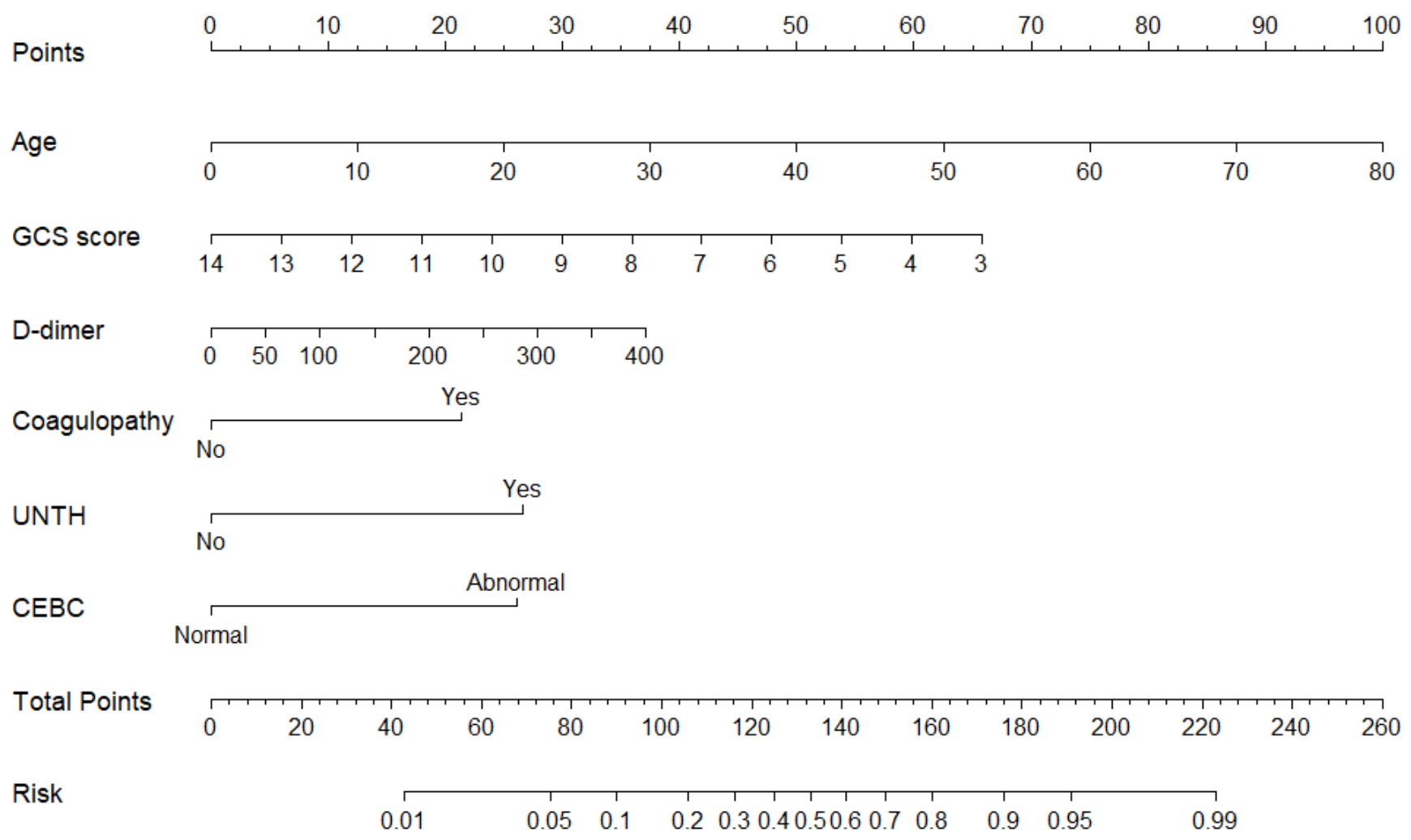

\section{Figure 2}

The nomogram for predicting 1-year mortality unfavorable outcome in TBI patients undergoing DC. The total points are calculated as the sum of the individual score of 6 variables included in the nomogram. UNTH: use of noradrenaline to treat hypotension; CEBC: completely effaced basal cisterns. 


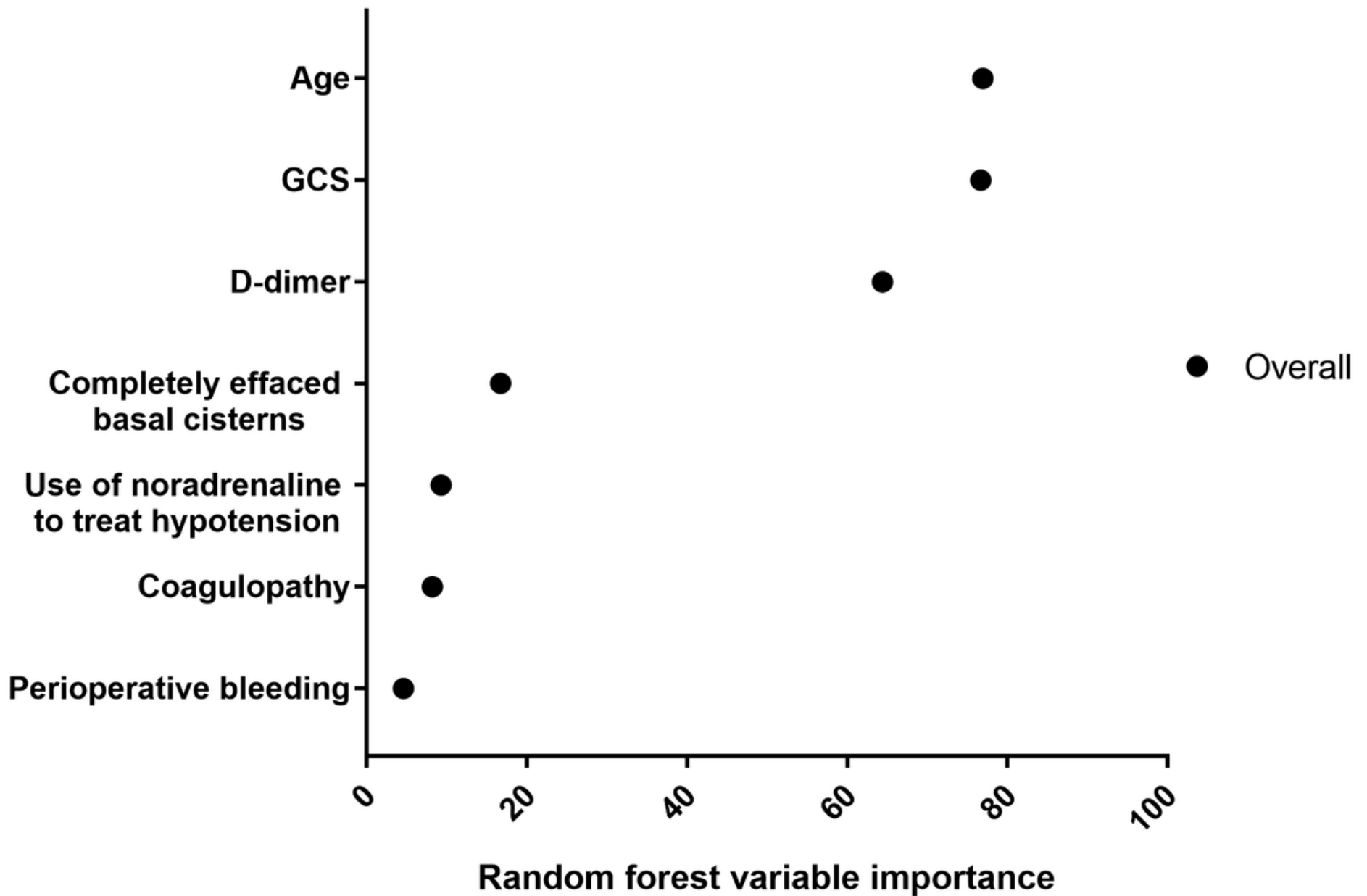

Figure 3

Variable importance measures for each predictor of 1-year mortality derived from random forest. 


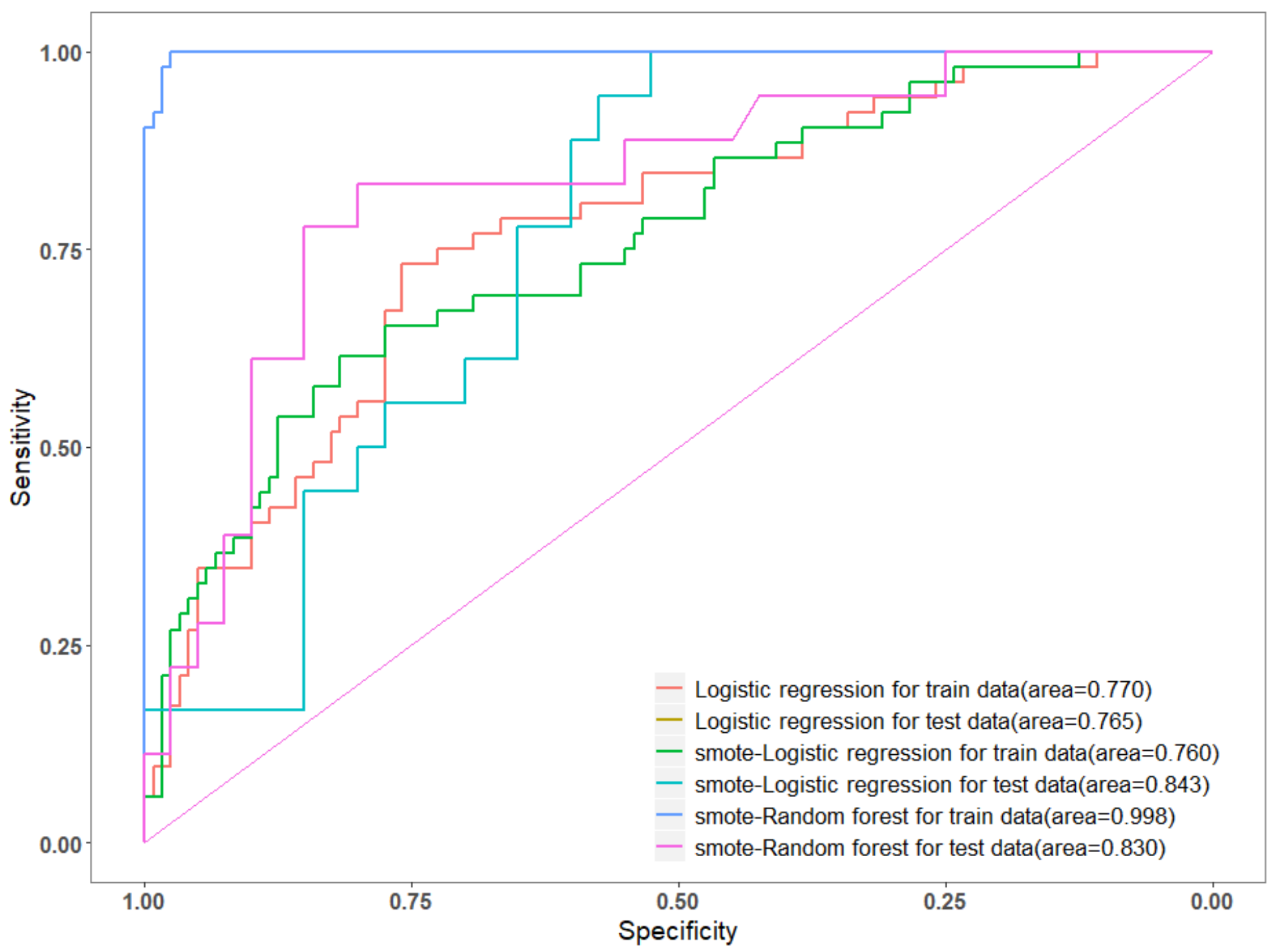

Figure 4

Receiver operating characteristic curves for 1-year mortality prediction in train sample and test sample.

\section{Supplementary Files}

This is a list of supplementary files associated with this preprint. Click to download.

- Table2.xlsx

- Table1.xlsx

- Table3.xlsx 